\title{
A FRAMEWORK TO SIMULTANEOUSLY EXPLAIN TINY NEUTRINO MASS AND HUGE MISSING MASS PROBLEM OF THE UNIVERSE
}

\author{
YASAMAN FARZAN ${ }^{1}$ \\ ${ }^{1}$ School of Physics, Institute for Research in Fundamental Sciences (IPM) \\ Tehran, PO. Box 19395-5531, Iran \\ yasaman@theory.ipm.ac.ir
}

\begin{abstract}
Recently a minimalistic scenario has been developed to explain dark matter and tiny but nonzero neutrino masses. In this scenario, a new scalar called SLIM plays the role of the dark matter. Neutrinos achieve Majorana mass through a one-loop diagram. This scenario can be realized for both real and complex SLIM. Simultaneously explaining the neutrino mass and dark matter abundance constrains the scenario. In particular for real SLIM, an upper bound of a few MeV on the masses of the new particles and a lower bound on their coupling are obtained which make the scenario testable. The low energy scenario can be embedded within various $S U(2) \times U(1)$ symmetric models. I shall briefly review the scenario and a specific model that embeds the scenario, with special emphasis on the effects in the charged Kaon decay which might be observable at the KLOE and NA62 experiments.
\end{abstract}

\section{INTRODUCTION}

The standard models of particles and cosmology have been unexpectedly successful in explaining various observed phenomena; however, there are two perplexing puzzles that are still unsolved: tiny but nonzero neutrino masses and the nature of dark matter. A considerable number of models beyond the Standard Model (SM) have been developed that address each of these puzzles but attempts to simultaneously explain them have only recently been started. In this letter, we shall review an economic scenario proposed in Ref. [1] which simultaneously explains the neutrino mass and dark matter puzzles. In this scenario, neutrinos acquire mass at one-loop level and the Dark Matter (DM) particles are produced thermally. The information on the neutrino mass matrix combined with the information on the dark matter abundance constrain the scenario and in most cases, make it testable.

The scenario is based on an effective low energy Lagrangian which has to be embedded within an electroweak symmetric model. The features of scenario show a guiding path to build models that embed the scenario. In Refs. [2, 3], two distinct models have been developed that embed the scenario. We review the model introduced in Ref. [2] and discuss its predictions. The model inherits the testability from the scenario. Moreover, the new fields added to make the model $S U(2) \times U(1)$ invariant cause observable effects in the LHC as well as in the low energy experiments searching for lepton flavor violation such as $\mu \rightarrow e \gamma$ searches. Since the model is very economic, as we shall see the information from these various low energy and high energy experiments can provide a possibility for cross check of the model.

The letter is organized as follows. In section $\amalg$ we introduce the content of the scenario and discuss the neutrino mass production and DM annihilation. In section ШII, we review the observable effects in the meson decay, core collapse supernova explosion and indirect DM searches with special emphasis on the testability of the model. In section IV] we review the model introduced in Ref. [2] and review the predictions of the model for the LHC and lepton flavor violating rare decays. In section】 we summarize the results and discuss the prospects.

\section{THE SCENARIO}

The scenario requires adding only the following particles to the SM: (i) a scalar which we call it SLIM: $\phi$; (ii) Two or more right-handed Majorana neutrinos, $N_{i}$ with mass term

$$
\frac{m_{N_{i}}}{2} N_{i}^{T}\left(i \sigma_{2}\right) N_{i}
$$

These new particles couple to the SM neutrinos through

$$
\mathcal{L}=g_{i \alpha} \phi \bar{N}_{i} v_{\alpha}
$$

Of course, such a coupling not being invariant under the $S U(2) \times U(1)$ symmetry is only a low energy effective coupling and at higher energies, as we shall discuss in section IV has to be augmented to become electroweak symmetric. A $Z_{2}$ symmetry under which only new particles are odd is imposed which forbids a Dirac mass term of type $\bar{N}_{i} v_{\alpha}$ and makes the lightest new particle stable and a suitable candidate for DM. We take $\phi$ to be the lightest new particle and therefore the DM candidate. The scenario can be realized both for cases that the scalar is real and complex. Let us study them one by one. 


\section{A. Real SLIM}

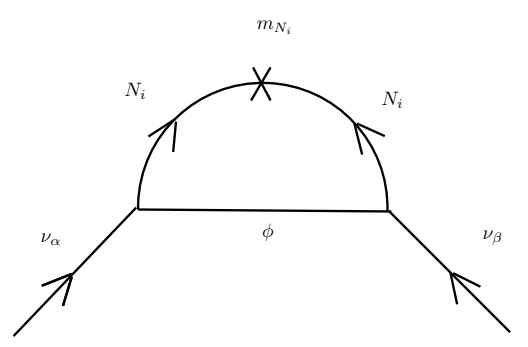

FIG. 1: Neutrino mass diagram

Real SLIM cannot carry lepton number. Neither do $N_{i}$ which are of Majorana type. Thus, the coupling in Eq. (1) violates lepton number conservation. Within this scenario, neutrinos through the one-loop diagram shown in Fig. 11acquire Majorana masses that can be written as follows

$$
\left(m_{v}\right)_{\alpha \beta}=\sum_{i} \frac{g_{i \alpha} g_{i \beta}}{16 \pi^{2}} m_{N_{i}}\left(\log \frac{\Lambda^{2}}{m_{N_{i}}^{2}}-\frac{m_{\phi}^{2}}{m_{N_{i}}^{2}-m_{\phi}^{2}} \log \frac{m_{N_{i}}^{2}}{m_{\phi}^{2}}\right),
$$

where $\Lambda$ is the ultraviolet cutoff of the effective coupling which is the electroweak scale. The neutrino mass matrix can in general be parameterized as

$$
m_{v}=U \cdot \operatorname{Diag}\left[m_{1}, m_{2} e^{2 i \gamma_{2}}, m_{3} e^{2 i \gamma_{3}}\right] U^{T},
$$

where $U$ is the famous PMNS mixing matrix and $\gamma_{2}$ and $\gamma_{3}$ are Majorana CP-violating phases. To accommodate the neutrino flavor structure, the coupling has to have the following structure:

$$
g=\operatorname{Diag}\left(X_{1}, \ldots, X_{n}\right) \cdot O \cdot \operatorname{Diag}\left(\sqrt{m_{1}}, \sqrt{m_{2}} e^{i \gamma_{2}}, \sqrt{m_{3}} e^{i \gamma_{3}}\right) U^{T},
$$

where $n$ is the number of Majorana neutrinos and

$$
X_{i}=4 \pi\left(\frac{1}{m_{N_{i}}}\right)^{1 / 2}\left(\log \frac{\Lambda^{2}}{m_{N_{i}}^{2}}-\frac{m_{\phi}^{2}}{m_{N_{i}}^{2}-m_{\phi}^{2}} \log \frac{m_{N_{i}}^{2}}{m_{\phi}^{2}}\right)^{-1 / 2},
$$

where $O$ is an arbitrary $n \times 3$ matrix that satisfies $O^{T} \cdot O=\operatorname{Diag}(1,1,1)$. Notice that for $n=3$, this means $O$ is an arbitrary orthogonal matrix. To accommodate the neutrino data with two nonzero mass eigenvalues, at least two nonzero right-handed neutrinos are needed. With only two right-handed neutrinos, one of the mass eigenvalues will vanish and the neutrino mass scheme will be hierarchical.

In this scenario, the DM in the early universe is thermally produced so its abundance is given by the inverse of its annihilation cross-section; see Ref. [4]. The main annihilation modes are lepton number violating ones $\phi \phi \rightarrow v v, \bar{v} \bar{v}$ which proceed via a $t$-channel lepton number violating right-handed neutrino exchange $(\langle N N\rangle)$ :

$$
\left\langle\sigma\left(\phi \phi \rightarrow v_{\alpha} v_{\beta}\right) v\right\rangle=\left\langle\sigma\left(\phi \phi \rightarrow \bar{v}_{\alpha} \bar{v}_{\beta}\right) v\right\rangle=\frac{1}{4 \pi}\left|\sum_{i} \frac{g_{i \alpha} g_{i \beta} m_{N_{i}}}{m_{\phi}^{2}+m_{N_{i}}^{2}}\right|^{2} .
$$

To account for the observed DM abundance, the annihilation cross section has to be of order of $10^{-36} \mathrm{~cm}^{2}$. Setting $\left\langle\sigma_{t o t} \nu\right\rangle$ equal to this value and inserting the observed neutrino masses, we find that the lighter right-handed neutrinos dominating the DM annihilation should have a mass in the range

$$
O(1) \mathrm{MeV} \lesssim m_{N_{1}} \lesssim 10 \mathrm{MeV} .
$$

The rest of $N_{i}$ can be heavier. To prevent the decay of the SLIM, it has to be lighter than $N_{1}$. From this consideration, we do not obtain any lower bound on the SLIM mass. The large scale structure arguments impose a lower bound of a few $\mathrm{keV}$ on the SLIM mass; see Refs. [5-8]. According to Ref. [9], Nucleosynthesis imposes even a stronger lower bound of MeV so

$$
O(1) \mathrm{MeV} \lesssim m_{\phi}<M_{N_{1}} \lesssim 10 \mathrm{MeV}
$$


Thus, SLIM has to be in the MeV range. In fact, SLIM stands for Scalar as LIght as MeV. From Eq. (5), we find

$$
g_{1 \alpha} \simeq 10^{-3} \sqrt{\frac{m_{N_{1}}}{10 \mathrm{MeV}}}\left(\frac{\left\langle\sigma v_{r}\right\rangle}{10^{-26} \mathrm{~cm}^{3} / \mathrm{s}}\right)^{\frac{1}{4}}\left(1+\frac{m_{\phi}^{2}}{m_{N_{1}}^{2}}\right)^{\frac{1}{2}}
$$

which leads to

$$
3 \times 10^{-4} \lesssim g_{1 \alpha} \lesssim 10^{-3}
$$

Lightness of $N_{1}$ and $\phi$ and the lower bound on their coupling imply that the scenario is testable in low energy phenomena. We will discuss this in more detail in section III

\section{B. Complex SLIM}

A complex $\phi$ can be decomposed in terms of its real components as $\phi \equiv\left(\phi_{1}+i \phi_{2}\right) / \sqrt{2}$. In practice, we have now two scalars with couplings $g_{i \alpha} / \sqrt{2}$ and $i g_{i \alpha} / \sqrt{2}$. The mass term for $\phi$ can in general be written as

$$
\mathcal{V}_{m}=M^{2} \phi^{\dagger} \phi-\frac{m^{2} \phi \phi+\text { H.c. }}{2}
$$

Imposing CP symmetry on $\mathcal{V}_{m}$ implies that $m^{2}$ is real. In this case, $\phi_{1}$ and $\phi_{2}$ will be mass eigenstates with masses

$$
\begin{aligned}
& m_{\phi_{1}}^{2}=M^{2}-m^{2} \\
& m_{\phi_{2}}^{2}=M^{2}+m^{2} .
\end{aligned}
$$

Thus, for nonzero $\mathrm{m}^{2}$, there is a splitting between the masses. Neutrinos obtain Majorana masses through the one-loop diagram shown in Fig. 1. Diagrams with $\phi_{1}$ and $\phi_{2}$ propagating in the loops should be summed up. Since their couplings are the same up to a factor of $i$, the cutoff dependent parts of the contributions of $\phi_{1}$ and $\phi_{2}$ cancel each other and we arrive at the following formula

$$
\left(m_{v}\right)_{\alpha \beta}=\sum_{i} \frac{g_{i \alpha} g_{i \beta}}{32 \pi^{2}} m_{N_{i}}\left[\frac{m_{\phi_{1}}^{2}}{\left(m_{N_{i}}^{2}-m_{\phi_{1}}^{2}\right)} \ln \frac{m_{N_{i}}^{2}}{m_{\phi_{1}}^{2}}-\frac{m_{\phi_{2}}^{2}}{\left(m_{N_{i}}^{2}-m_{\phi_{2}}^{2}\right)} \ln \frac{m_{N_{i}}^{2}}{m_{\phi_{2}}^{2}}\right] .
$$

Notice that for $m_{\phi_{1}} \rightarrow m_{\phi_{2}}, m_{v}$ vanishes. This is understandable because at this limit (i.e., $m^{2} \rightarrow 0$ ), the Lagrangian becomes lepton number conserving by assigning a lepton number of -1 to $\phi$. As a result, in this limit, the lepton number violating Majorana mass, $m_{v}$, should vanish.

Without loss of generality, we can take $\phi_{1}$ to be lighter than $\phi_{2}$ and therefore a DM candidate. Since the couplings of $\phi_{1}$ are similar to real SLIM up to a factor of $\sqrt{2}$, the annihilation cross section of $\phi_{1}$ is given by Eq. (5) replacing $g$ with $g / \sqrt{2}$.

Unlike the real SLIM case, in the complex scenario, the right-handed neutrinos can be as heavy as the electroweak scale or even heavier. $\phi_{1}$ and $\phi_{2}$ can be heavy, too. For $m_{\phi_{1}} \gg 10 \mathrm{MeV}, \phi_{1}$ and $\phi_{2}$ will be quasi-degenerate and their coannihilation at freeze out has to be taken into account. Notice however that within this scenario, $\sigma\left(\phi_{1} \phi_{2} \rightarrow v v\right) \sim \sigma\left(\phi_{1} \phi_{1} \rightarrow \mathrm{vv}\right)$ so the overall picture will not be altered by coannihilation.

\section{PHENOMENOLOGICAL IMPLICATIONS OF THE SCENARIO}

The scenario, specially in the real SLIM mode, contains light particles with small (but bounded from below) coupling with $v$. As a result, we expect the scenario to have its imprint in various phenomena. One possibility is nucleosynthesis which as shown in Ref. [9] implies a lower bound of MeV on the SLIM mass. Different possibilities have been enumerated in Ref. [1]. In this section, we discuss the effects in particle decays, indirect searches by neutrino detectors as well as core collapse supernova explosion which are among the most promising observations to look for the SLIM.

\section{A. Light Meson and tau lepton decays}

Consider any of the particle decays within SM that produce a neutrino which appears as missing energy:

$$
A \rightarrow B+\mathrm{v}
$$


In the presence of the new coupling Eq. (1), along with this decay mode, $A$ can go through the following decay via a virtual $v$ exchange

$$
A \rightarrow B N_{i} \phi
$$

of course provided that the difference between $A$ mass and the sum of masses in $B$ is larger than the sum of the masses of $\phi$ and $N_{i}$. Remember that in case of real SLIM, we find $m_{\phi}<m_{N_{1}}<10 \mathrm{MeV}$ so decays such as $K(\pi) \rightarrow \mu(e) N_{1} \phi$ or $\tau \rightarrow \mu(e) \nu N_{1} \phi$ are possible. $\phi$ and $N_{i}$ will show up as missing energy. Thus, by comparing the measured value of $A \rightarrow B+$ missing energy with the SM prediction $\left|g_{i \alpha}\right|$ can be constrained. Such an analysis has not been performed for the SLIM model but similar analysis has extensively been carried out (see Refs. [10-13]) in the case of the Majoron couplings to neutrinos $\tilde{g}_{\alpha \beta} J v_{\alpha}^{c} v_{\beta}$ where $J$ is the Majoron which is a massless pseudoscalar. Such a coupling will also contribute to $A \rightarrow B+$ missing energy. As long as the sum of the masses of $N_{i}$ and $\phi$ is much smaller than the difference between the masses of $A$ and $B$, the bounds for Majoron also apply for the SLIM case. The strongest bounds come from the Kaon and tau decay for which this condition is satisfied.

The new SLIM interaction can give rise to new channels of $\left(K^{+} \rightarrow \mu^{+}+\right.$missing energy) and $\left(K^{+} \rightarrow e^{+}+\right.$missing energy) causing a deviation from the universality predicted by the SM:

$$
\begin{gathered}
\frac{\Gamma\left(K^{+} \rightarrow e^{+}+\text {missing energy }\right)}{\Gamma\left(K^{+} \rightarrow \mu^{+}+\text {missing energy }\right)}=\frac{\Gamma_{S M}\left(K^{+} \rightarrow e^{+} v_{e}\right)+\sum_{i} \Gamma\left(K^{+} \rightarrow e^{+} N_{i} \phi\right)}{\Gamma_{S M}\left(K^{+} \rightarrow \mu^{+} v_{e}\right)+\sum_{i} \Gamma\left(K^{+} \rightarrow \mu^{+} N_{i} \phi\right)} \\
\simeq \frac{\Gamma_{S M}\left(K^{+} \rightarrow e^{+} v_{e}\right)+\sum_{i} \Gamma\left(K^{+} \rightarrow e^{+} N_{i} \phi\right)}{\Gamma_{S M}\left(K^{+} \rightarrow \mu^{+} v_{e}\right)}
\end{gathered}
$$

By comparing the SM prediction with the most recent results from KLOE in Ref. [14], one can obtain

$$
\sum_{i}\left|g_{i e}\right|^{2}<10^{-5}
$$

where the sum runs over $N_{i}$ lighter than $K^{+}$. Notice that this bound is too weak to probe the range in Eq. (8). Another bound can be obtained by studying the spectrum of the muon produced in the Kaon decay. Of course, the spectrum of the muon in $K^{+} \rightarrow \mu^{+} N_{i} \phi$, being a three-body decay, will be considerably different from that in $K^{+} \rightarrow \mu^{+}+v_{\mu}$, being a two-body decay. An indirect way to extract the bound is to use the bound on $K^{+} \rightarrow \mu^{+} v_{\mu} v \bar{v}$. This four-body decay is allowed in the SM but the SM prediction is completely negligible and far below the present bound found by Ref. [16] which is

$$
\operatorname{Br}\left(K^{+} \rightarrow \mu^{+}+v_{\mu}+v+\bar{v}\right)<6 \times 10^{-6} .
$$

In Ref. [13], this bound is interpreted as the bound on the new modes of $\left(K^{+} \rightarrow \mu^{+}+\right.$missing energy). Such an interpretation is not of course completely accurate as the spectrum for three body decay and four body decays are not the same but tentatively it is acceptable. Taking the same approach, we find

$$
\sum_{i}\left|g_{i \mu}\right|^{2}<9 \times 10^{-5}
$$

Notice that this bound is based on the old LBL Bevatron data obtained in 1973. It seems if the recent KLOE data is analyzed to search for the $K^{+} \rightarrow \mu^{+} N_{i} \phi$ signal, a considerable part of the range in Eq. (8) can be probed. Moreover upcoming NA62 experiment at CERN might be sensitive to even smaller values of couplings. For a description of NA62 see Ref. [15]. Another bound which has been studied in Ref. [13] is the bound from the $\tau$ decay which in our scenario can be translated into

$$
\sum_{i}\left|g_{i \tau}\right|^{2}<10^{-1}
$$

In summary, the present bounds from meson and tau decays are too week to probe the range in Eq. (8); however, there is a hope that signals for this scenario already lie in the new data from KLOE.

\section{B. Supernova type II explosion}

In our scenario, SLIMs can be produced by neutrinos inside the supernova core. The produced SLIMs will undergo scattering on the neutrinos already present in the core. The scattering cross section in the outer core where the chemical potential of neutrinos vanishes is given by

$$
\sigma(\phi v \rightarrow \phi v) \sim \frac{g^{4} T^{2}}{4 \pi\left(T^{2}+m_{N_{i}}^{2}\right)^{2}}
$$


Taking the temperature to be of $T \sim 30 \mathrm{MeV}$, we find that the mean free path is $\left(\sigma n_{v}\right)^{-1} \sim 10 \mathrm{~cm}$ which is far smaller than the supernova core. This means SLIMs will be trapped inside the core and their contribution to cooling is only as a new single scalar mode diffuse out which can be well tolerated within the present observational and the supernova model uncertainties. Notice that in our scenario SLIMs do not interact with nuclei so the constraint in Ref. [17] does not apply to this scenario. For a more detailed discussion see Ref. [1].

It is also interesting to note that in the case of future supernova neutrino observations, one may be able to test this scenario by studying the neutrino energy spectra.

\section{Indirect DM searches}

In this scenario DM particles only interact with neutrinos so we do not expect any photon signal from their annihilation (neither prompt nor secondary). Moreover unlike WIMPs, SLIMs do not interact with nuclei in the Earth or Sun so they do not get trapped in them and we do not therefore expect a detectable neutrino flux from DM annihilation in the Sun or Earth center. However, the DM annihilation in the halo can result in a sizeable neutrino flux at neutrino detectors such as super-kamiokande. This effect has been studied in Ref. [18]. The present bound from super-kamiokande is too weak to be sensitive to $\left\langle\sigma_{\text {tot }} v\right\rangle \sim 10^{-36} \mathrm{~cm}^{2}$. However, as shown in Ref. [18], with LENA (described in Ref. [19]) or a megaton water detector doped with Gd, it will be possible to probe the scenario for $m_{\phi} \sim 20-30 \mathrm{MeV}$. Remember that such a mass range is above the upper bound for real SLIM and can be realized only for a complex SLIM.

\section{MODELS EMBEDDING THE SCENARIO}

In Ref. [1], several routes have been suggested to embed the scenario in an electroweak invariant model. As we saw in section [I] in the case of real SLIM, at least one of the right-handed neutrinos has to be lighter than $10 \mathrm{MeV}$. As a result, it should be an electroweak singlet to avoid the bounds from the invisible decay width of the $Z$ boson. However, in the case of complex SLIM, $N_{i}$ can be heavier than $m_{Z} / 2$ and can therefore be a component of an electroweak multiplet.

The model introduced in Ref. [3] can be considered a realization of a model embedding the complex SLIM scenario. On the other hand, the model in Ref. [2] is an example for embedding real SLIM scenario. In the following, we describe the minimalistic model introduced in Ref. [2] that has interesting predictions for the LHC and the lepton flavor violating rare decays.

To make the coupling in Eq. (1) $S U(2) \times U(1)$ invariant, $\phi$ is promoted to an electroweak doublet $\Phi=\left(\phi^{0} \phi^{-}\right)$. Of course, in this case $\phi^{0}$ which carries nonzero hypercharge has to be complex and can be decomposed as $\left(\phi_{1}+i \phi_{2}\right) / \sqrt{2}$ in terms of real scalars, $\phi_{1}$ and $\phi_{2}$. With a coupling of form $\operatorname{Re}\left[\left(H^{T}\left(i \sigma_{2}\right) \Phi\right)^{2}\right]$, the vacuum expectation value of the Higgs leads to a small splitting between the $\phi_{1}$ and $\phi_{2}$ components which is what desired in the complex SLIM model. At first sight, it seems that with this minimalistic content, one can embed complex SLIM scenario but this is not the case: $\phi_{1}$ and $\phi_{2}$, being the components of an electroweak doublet have to be heavier than about $80 \mathrm{GeV}$ to satisfy the present bounds in Refs. [20-22]. On the other hand as we saw in section IIB, within this scenario the splitting between $m_{\phi_{1}}^{2}$ and $m_{\phi_{2}}^{2}$ has to be smaller than $(20 \mathrm{MeV})^{2}$. That is $\phi_{1}$ and $\phi_{2}$ will be quasi-degenerate and having coupling to the $Z$ boson, they can coannihilate in the early universe leading to a too low dark matter density (see Ref. [23]). In Ref. [2], a new scalar $\eta$, singlet under the electroweak transformation, is added which mixes with the neutral component of $\Phi$. The DM candidate, SLIM, is a mixture of $\eta$ and $\phi_{1}$ with mixing angle $\alpha$. Neutrinos obtain mass through one loop diagram. In fact, the masses of the components of $\Phi$ play the role of cut-off in Eq. (2). The model inherits the features of the real SLIM scenario (i.e., lower bound on the coupling and an upper bound on the masses of the SLIM and $N_{1}$ ) so the model is testable by low energy high precision experiments such as the Kaon decay measurements. Moreover, the charged component of $\Phi$ leads to observable effects on $(g-2)_{\mu}$ and lepton flavor violating rare decays. The effects on $(g-2)_{\mu}$ is two orders of magnitudes below the present sensitivity but as shown in Ref. [2], the present bound on $\mu \rightarrow e \gamma$ already constrains a part of the parameter space. The MEG experiment searching for $\mu \rightarrow e \gamma$ can further constrain the model or hopefully find a hint. If the masses of the components of $\Phi$ are not too high, they can be copiously produced via the electroweak interaction at the LHC. Their subsequent decays can be driven by coupling in Eq. (1); in particular, $\Gamma\left(\phi^{-} \rightarrow \ell_{\alpha}^{-} N_{i}\right) \propto\left|g_{i \alpha}\right|^{2}$. That is by studying the decay modes of $\phi^{-}$, it is possible to derive the flavor structure of $\left|g_{i \alpha}\right|$. Remember that $g_{i \alpha}$ is the same coupling that determines the neutrino mass matrix as well as the rates of lepton flavor violating decays such as $\mu \rightarrow e \gamma, \tau \rightarrow \mu \gamma$ and $\tau \rightarrow e \gamma$. That is, information on new couplings $g_{i \alpha}$ can be derived by all these three methods which provide a way for cross check and test of the model.

In Ref. [24], the discovery potential of the LHC is explored taking into account the detailed background. The production cross section is of order of a few hundred $f b$ for $m_{\phi^{-}}<150 \mathrm{GeV}$. It is shown in Ref. [24] that by employing the state-of-the-art cuts, signal significance can well exceed $5 \sigma$ for the $14 \mathrm{TeV}$ run of the LHC with $30 \mathrm{fb}^{-1}$. The possibility of deriving the flavor structure of the coupling $g_{i \alpha}$ is also discussed. 


\section{SUMMARY AND DISCUSSION}

In this letter, we have reviewed a scenario that links DM and neutrino masses. The scenario is based on a scalar, $\phi$, coupled to the SM left-handed neutrinos and two or more right-handed Majorana neutrinos: $g_{i \alpha} \bar{N}_{i} v_{\alpha} \phi$. The scalar plays the role of the DM and is stabilized via a $Z_{2}$ symmetry which also forbids a Dirac mass for neutrinos. DM particle in this scenario mainly annihilate into $v V$ and $\bar{v} \bar{v}$. This would lead to a diffuse flux from the DM halo. The present upper bound from super-kamiokande is too weak to probe the scenario which is based on the assumption of thermal production of the DM in the early universe and therefore $\left\langle\sigma_{t o t} v\right\rangle \simeq 10^{-36} \mathrm{~cm}^{2}$. In future, a LENA type detector or a megaton water detector doped with Gd can probe the model for DM mass around 20 to $30 \mathrm{MeV}$. The scenario can be realized in two cases: (i) real $\phi$; (ii) complex $\phi$.

In the real SLIM case, we found $m_{\phi}<m_{N_{1}}<10 \mathrm{MeV}$ and $3 \times 10^{-4} \lesssim g_{1 \alpha} \lesssim 10^{-3}$. As a result, new missing energy modes are predicted at kaon and tau lepton decays. The present bounds are too weak to probe the scenario but eventual improvement can test the model because the upper bound on $m_{\phi}$ and $m_{N_{1}}$ combined with the lower bound on $g_{1 \alpha}$ imply a lower bound on the rates of the new decay modes. A re-analysis of KLOE data with the aim of searching for $K^{+} \rightarrow \mu^{+} \phi N_{1}$ and $K^{+} \rightarrow e^{+} \phi N_{1}$ will be most promising. Upcoming NA62 experiment might be even more sensitive. Real SLIM, being light, can be also produced via neutrinos in a supernova core. The produced SLIM will be trapped in the core but will eventually diffuse out and contribute to the cooling of the supernova core. This new cooling mode can be tolerated within the present uncertainties but in future if the observational and supernova model uncertainties are reduced, the presence of this new mode can be tested. The SLIM emission can also distort the spectrum of the emitted neutrinos.

Complex SLIM can be as heavy as the electroweak scale but the mass splitting between the CP-odd and -even components (i.e., $\left.\left|m_{\phi_{1}}^{2}-m_{\phi_{2}}^{2}\right|\right)$ must be between $(10 \mathrm{MeV})^{2}$ to $(20 \mathrm{MeV})^{2}$.

We discussed the possibility of embedding the scenario within a $S U(2) \times U(1)$ invariant model. We focused on the model developed in Ref. [2] which embeds the real SLIM scenario. In addition to being falsifiable by low energy experiments, the model predicts observable effects for lepton flavor violating rare decays, $(g-2)_{\mu}$ and the LHC. In particular, a hint for new physics in running searches at MEG is expected. The model has certain predictions for the LHC (see Ref. [24]). It is in principle possible to measure $g_{i \alpha}$ at the LHC. This is the same coupling determining the neutrino mass matrix and lepton flavor violating rare decays and the flavor pattern of new modes of $K^{+} \rightarrow \ell_{\alpha}^{+}+$missing energy. Thus, there is a possibility of cross-checking the model by low energy and high energy experiments.

It might be possible to augment the scenario to account for the baryon asymmetry of the universe through the mechanism introduced in Ref. [25].

\section{Acknowledgments}

I would like to thank Celine Boehm, Thomas Hambye, Sergio Palomares-Ruiz and Silvia Pascoli. I would also like to specially thank Prof G. Isidori for useful remarks. I am also grateful to the organizers of Planck 2009 meeting, where this talk was presented, for their hospitality.

[1] C. Boehm, Y. Farzan, T. Hambye, S. Palomares-Ruiz and S. Pascoli, Phys. Rev. D 77, 043516 (2008) [arXiv:hep-ph/0612228].

[2] Y. Farzan, Phys. Rev. D 80, 073009 (2009) |arXiv:0908.3729 [hep-ph]].

[3] Y. Farzan, S. Pascoli and M. A. Schmidt, arXiv:1005.5323 [hep-ph].

[4] B. W. Lee and S. Weinberg, Phys. Rev. Lett. 39, 165 (1977).

[5] M. Viel, J. Lesgourgues, M. G. Haehnelt, S. Matarrese and A. Riotto, Phys. Rev. D 71, 063534 (2005) |arXiv:astro-ph/0501562];

[6] U. Seljak, A. Makarov, P. McDonald and H. Trac, Phys. Rev. Lett. 97, 191303 (2006) |arXiv:astro-ph/0602430];

[7] M. Viel, J. Lesgourgues, M. G. Haehnelt, S. Matarrese and A. Riotto, Phys. Rev. Lett. 97, 071301 (2006) [arXiv:astro-ph/0605706];

[8] M. Miranda and A. V. Maccio, arXiv:0706.0896 [astro-ph].

[9] P. D. Serpico and G. G. Raffelt, Phys. Rev. D 70, 043526 (2004) |arXiv:astro-ph/0403417|.

[10] D. I. Britton et al., Phys. Rev. D 49, 28 (1994).

[11] V. D. Barger, W. Y. Keung and S. Pakvasa, Phys. Rev. D 25, 907 (1982).

[12] G. B. Gelmini, S. Nussinov and M. Roncadelli, Nucl. Phys. B 209, 157 (1982).

[13] A. P. Lessa and O. L. G. Peres, Phys. Rev. D 75, 094001 (2007) [arXiv:hep-ph/0701068].

[14] F. Ambrosino et al. [KLOE Collaboration], Eur. Phys. J. C 64, 627 (2009) [Erratum-ibid. 65, 703 (2010)] [arXiv:0907.3594][hep-ex]].

[15] http://na62.web.cern.ch/NA62/Home/Home.html

[16] C. Y. Pang, R. H. Hildebrand, G. D. Cable and R. Stiening, Phys. Rev. D 8, 1989 (1973).

[17] P. Fayet, D. Hooper and G. Sigl, Phys. Rev. Lett. 96 (2006) 211302 [arXiv:hep-ph/0602169].

[18] S. Palomares-Ruiz and S. Pascoli, Phys. Rev. D 77, 025025 (2008) [arXiv:0710.5420 [astro-ph]].

[19] T. Marrodan Undagoitia et al., J. Phys. Conf. Ser. 120, 052018 (2008). 
[20] [LEP Higgs Working Group for Higgs boson searches and ALEPH Collaboration an], arXiv:hep-ex/0107031

[21] G. Abbiendi et al. [OPAL Collaboration], Eur. Phys. J. C 32, 453 (2004) [arXiv:hep-ex/0309014].

[22] G. Abbiendi et al. [OPAL Collaboration], arXiv:0812.0267[ [hep-ex].

[23] R. Barbieri, L. J. Hall and V. S. Rychkov, Phys. Rev. D 74, 015007 (2006) [arXiv:hep-ph/0603188].

[24] Y. Farzan and M. Hashemi, arXiv:1009.0829 [hep-ph].

[25] E. K. Akhmedov, V. A. Rubakov and A. Y. Smirnov, Phys. Rev. Lett. 81, 1359 (1998) arXiv:hep-ph/9803255]. 